\title{
Polysèmes
}

Revue d'études intertextuelles et intermédiales

\section{Transpositions et deuil dans « Rose-Coloured Teacups » d'A.S. Byatt}

\section{Pascale Tollance}

\section{(2) OpenEdition}

\section{Journals}

Édition électronique

URL : http://journals.openedition.org/polysemes/687

DOI : $10.4000 /$ polysemes. 687

ISSN : 2496-4212

Éditeur

SAIT

Édition imprimée

Date de publication : 1 janvier 2012

Pagination : 285-310

ISSN : 0999-4203

\section{Référence électronique}

Pascale Tollance, «Transpositions et deuil dans «Rose-Coloured Teacups » d'A.S. Byatt », Polysèmes [En ligne], 12 | 2012, mis en ligne le 01 mars 2015, consulté le 21 avril 2019. URL : http:// journals.openedition.org/polysemes/687; DOI : 10.4000/polysemes.687

Ce document a été généré automatiquement le 21 avril 2019.

Polysèmes 


\title{
Transpositions et deuil dans « Rose- Coloured Teacups » d'A.S. Byatt
}

\author{
Pascale Tollance
}

1 Cette brève nouvelle d'A.S. Byatt ${ }^{1}$ nous présente une femme seule, travaillant en silence à un curieux ouvrage, puisqu'elle compose en imagination un tableau énigmatique, dont on comprendra après coup qu'il est à la fois transposition et recomposition de fragments de vie épars. Le tableau imaginaire s'organise en deux pans, l'un ouvrant la nouvelle, l'autre la clôturant, séparés par un incident au présent qui interrompt la rêverie du personnage et en même temps ravive un incident passé. Dès le départ le lecteur, largement sollicité, se voit entraîné dans un parcours où il est amené à repérer les passages et les reprises d'une partie du texte à l'autre pour tenter de donner un sens au tableau initial. Mais au moment où ce tableau est décrit une seconde fois, alors que le texte semble sur le point de se boucler, la nouvelle déplace et relance complètement le travail de lecture. Jusque-là, la composition imaginaire, présentée explicitement comme un travail de deuil entamé depuis la mort de la mère, nous montrait trois femmes en train de prendre le thé par une belle après-midi d'été dans la chambre d'un college de Cambridge. La fin de la nouvelle fait surgir aux marges du tableau, par une porte qui s'ouvre, un « him » qui a de quoi dérouter le lecteur. À ce point la vision s'évanouit, il est impossible de voir "plus loin»; la dernière phrase nous présente Veronica, l'auteur du tableau, s'apprêtant à recommencer son ouvrage du début.

2 Si le tableau imaginaire constitue un travail de deuil, celui-ci se voit à plus d'un titre différé par la fin de la nouvelle. Le travail de transposition auquel se livre Veronica se donne comme un effort pour poser une vision qui, tout en prenant une définition très précise, ne se pose pas : exercice sans cesse à reprendre, le tableau est aussi une œuvre fuyante dont on ne pourra arrêter définitivement le sens. La fin de la nouvelle semble alors entraver l'éventuelle transformation à laquelle la transposition pourrait œuvrer transformation par laquelle l'attachement manifesté à un objet premier pourrait déjà être une forme de détachement. Selon Freud, pour que le travail de deuil s'effectue, "l'existence de l'objet est continuée psychiquement. Chacun des souvenirs et des attentes, pris un à un, dans lesquels la libido était rattachée à l'objet, est mis en position, 
surinvesti, et sur chacun est effectué le détachement de la libido $»^{2}$. Ce mouvement conjoint de surinvestissement et de détachement s'exprime à travers diverses tensions dans la vision : si le tableau transpose et accomplit un rêve originel d'arrêter le temps, il possède aussi toute l'ambiguïté d'une nature morte. En même temps qu'il tente de préserver et de réparer un objet premier, l'ouvrage ne dissimule pas sa nature de bricolage, découpant et mettant en pièces autant qu'il rapièce l'original. Inversement, la composition de Veronica n'est qu'un arrangement précaire : tracée sur mesure, maîtrisée dans les moindres détails, elle est aussi travaillée par un sens qui échappe à celle qui voit ; le tableau garde ses zones d'ombres, reste ouvert sur un ailleurs d'où surgira l'apparition finale qui le décentre. Ce décentrement passe par l'insertion d'une sorte de tableau dans le tableau, à ceci près que celui-ci n'arrive pas vraiment à prendre forme et demeure une vision fugitive devant laquelle le texte s'éclipse. Le passage du visuel au textuel rencontre à ce point une résistance qui nous amène à interroger la dynamique d'ensemble d'un texte qui se donne pour une grande partie comme la transcription d'une image virtuelle. Alors que le travail de deuil de Veronica semble buter sur la force d'une présence qui s'impose au regard, on se demandera si le texte doit pour sa part renoncer ou non à faire partager un pouvoir qui serait propre à l'image.

\section{Ressusciter ou faire taire les morts}

3 Parmi tous les déplacements qui sont à l'œuvre dans la construction imaginaire du tableau, il en est un qui semble primordial : c'est celui par lequel la vision fugace d'une chambre à Cambridge, "two low chairs and a bed under a window» (37), se voit transportée sur le tableau de Veronica pour en devenir la matrice. Quelles que soient les variations qui interviennent au fil des reprises, c'est toujours à l'intérieur de cette chambre qu'elles prendront place. Veronica y installe trois femmes autour d'une tasse de thé : sa mère, désignée explicitement comme telle dans le deuxième pan de la description du tableau; une autre femme que Veronica doit s'empêcher de voir comme une femme âgée et qui pourrait être sa grand-mère; et enfin, une troisième femme, que rien ne permet d'identifier, mais qui pourrait être la représentante d'une troisième génération, et donc éventuellement Veronica elle-même. On apprend au cours de la nouvelle que la chambre est en partie un souvenir de Veronica étudiante, une vision saisie au détour d'un couloir alors que la jeune fille se promène dans la partie du collège où a résidé sa mère trente ans plus tôt. La chambre se trouve associée à un fantasme maternel que la jeune fille a alors manqué d'accomplir : "It had been a fantasy that Veronica would sit in the same chairs, in the same sunlight and drink from the same cups » (37).

4 En même temps qu'un lieu, c'est déjà un rêve qui se trouve donc transposé sur le tableau imaginaire. L'étudiante ayant en réalité déçu l'attente maternelle, la femme mûre tenterait après coup d'y répondre imaginairement en traduisant à la lettre le fantasme qu'elle prête à sa mère. Tout l'effort consisterait à aménager une vision première afin d'accomplir mot pour mot un désir. Pour ce faire, et notamment afin de faire boire les trois femmes dans les tasses en question, il est alors nécessaire non seulement de rajeunir deux des personnages, mais aussi d'effacer un chapitre clé du passé, évoqué au centre de la nouvelle : l'épisode où Veronica et sa mère ouvrent un carton et découvrent des éclats de porcelaine à la place des exquises tasses à thé que la fille a reçues de sa mère. La reprise s'accompagne d'un travail de réparation, matérialisé par la présence des tasses mais aussi par la description méticuleuse et détaillée de la scène qui étoffe la vision. 
Chacune des phrases qui posent un à un les éléments du tableau s'apparente en effet à un geste exact et précis, et témoigne d'un soin qui rachète la négligence passée lors de l'emballage précipité des tasses. La composition vient alors former un écrin à l'intérieur duquel l'objet précieux sera mis à l'abri des outrages du temps, mais aussi un refuge, «a safe place » (ce sont les derniers mots de la nouvelle) où Veronica peut se retirer. En posant une vision fugitive et le rêve qui l'accompagne d'arrêter le temps, la transposition permet aussi de poser et d'encadrer un objet « surinvesti ». Mais la reprise obsessionnelle du tableau suggère une fixation, une inertie qui semble obstruer le travail du deuil et ne laisse pas envisager la perspective d'un détachement. Le «tableau-refuge » serait-il alors pour Veronica une façon de conjurer une menace qui n'est autre que la vie?

5 Celle-ci prend une forme très voyante et très bruyante à l'extérieur du tableau : elle est incarnée par Jane, la fille de Veronica, qui interrompt la rêverie de sa mère, « peremptory and wailing » (34), pour lui annoncer que la machine à coudre ancestrale vient de rendre l'âme. Comme Veronica quelques années plus tôt, Jane a sauvagement mis en pièces le précieux objet familial. Mais, peu portée au remords, elle abandonne bien vite sa mère au milieu du chantier pour retourner dans le tourbillon de la vie. Tandis que Veronica reconstruit en silence ses tasses de porcelaine rose, Jane cultive un art plus éphémère, celui de la coiffure et du maquillage : sa chevelure, " her erect and lacquered hair ", est décrite comme "a jagged work of art » (34), œuvre d'art quelque peu agressive qui fait des cheveux qui se dressent autant de lames ou d'éclats tranchants; sa bouche, quant à elle, est peinte, « painted a glossy magenta » (35), couleur primaire qui contraste avec le rose délicat des tasses. Tout l'univers de Jane est « loud » et cela pas seulement en raison des couleurs qu'elle affectionne; sa vie trépidante, débordante (« which flowed and overflowed from house to house ») est décrite comme «loud with rock, pungent with illegal smoke, vigorous-voiced » (34).

6 En retrait de ce présent trop bruyant et trop criard, Veronica construit à l'intérieur d'un cadre un univers à la fois immobile et silencieux, «still ». Toutefois, et c'est là que son travail se charge d'ambiguïté, les voix qu'il faut faire taire ne sont pas seulement à l'extérieur, mais aussi à l'intérieur du tableau. Face au spectacle de la machine éventrée, Veronica, prise de rage, est sur le point de hurler et s'entend de façon anticipée : « [She] heard in her mind's ear a preliminary ghost of her own voice about to embark on a howling plaint, how could you... » (36). Le fantôme de cette voix est de fait un souvenir, le souvenir de sa propre mère : "And abruptly remembered her own mother's voice in the 1950's, unrestrained, wailing, interminable, how could you, how could you » (36). Le tableau imaginaire que construit Veronica de façon répétée depuis la mort de sa mère est alors explicitement associé au désir de ne plus entendre cette voix :

It was a curious form of mourning, but compulsive, and partly comforting. It seemed to be all she was capable of. The force of her mother's rage against the house and the housewifery that trapped her and, by extension, her clever daughters, who had all partly evaded that trap, precluded wholehearted mourning. The silence of her absence was like coming in out of a storm. Or like the silence of that still little room, in its bright expectancy, one or any afternoon in the late 1950's. (36)

7 Si le tableau constitue une "curieuse forme de deuil ", c'est alors peut-être moins parce qu'il est entravé par une fixation mélancolique que parce qu'il fait taire cette mère hurlant son refus de voir ses filles s'éloigner d'elle. L'idée que le tableau transposerait le désir maternel d'un temps suspendu devient donc problématique face à la hantise de la reproduction du même qui semble toute aussi forte que l'angoisse de la cassure. Le 
souvenir de la colère maternelle est ce qui empêche Veronica de laisser à son tour libre cours à sa colère : "She could not reproduce that fury against Jane » (36). Par ailleurs, il est dit que Veronica n'a pas cherché à faire asseoir ses filles dans le même fauteuil qu'elle, au moment où l'ainée est entrée à l'université : « No one steps in the same river twice. Jane's elder sister, Veronica's elder daughter, had also gone to the college, and Veronica, forewarned, had watched her assert her place in it, her here and now » (37).

En se chargeant de significations croisées, la vision ne devient pas simplement ambivalente mais aussi incertaine et flottante. Transposition littérale, le tableau l'est dans la mesure où il reprend tels quels les éléments d'une phrase, mais le signifiant est ici conservé aux dépens même du signifié qui lui était accolé. Plus qu'une translation dans l'espace, la transposition consiste en la transformation d'un lieu clos en un espace ouvert. Dans le fantasme que Veronica prête à sa mère, la chambre de Cambridge se pose comme une image qui tente de contredire une autre image, figée, celle de la rivière figurant l'écoulement du temps: "No one steps in the same river twice». Dans le tableau imaginaire, la petite chambre lumineuse, détachée de son ancrage initial, devient justement l'espace où tout déplacement, $\mathrm{y}$ compris la reprise du même, introduit un écart. Le topos de la mutabilité des choses se trouve réactivé dans le lieu même qui tentait de le nier. En même temps, à l'image figée, au lieu commun, est substitué un espace singulier avec son fonctionnement propre. L'enjeu de la vision est bien de poser et de positionner les choses si l'on prête attention au soin avec lequel est décrite la place de chaque personnage et de chaque objet dans le tableau. En même temps, la transposition met en branle le sens, engendre un mouvement qui fait qu'au sein même du tableau quelque chose ne se pose pas.

\section{Dédoublement}

Rêve d'immobilité et de silence, de «stillness », la vision de Veronica ne cesse pourtant de bouger. Cela se traduit d'abord par la façon dont le tableau parvient à associer immobilité et mouvement, bruit et silence. Des trois femmes, il nous est dit: "They were young women, full of energy; this could be seen in the quick, alert turns of the heads, the movements of hand to mouth, carrying a cigarette in a long holder, a rose-coloured teacup » (33). Un peu plus bas, ces femmes se mettent à parler : "The women talked to each other. They were waiting for someone. She could not hear their conversation or their occasional laughter » (34). La vision suspend la vie sans la figer comme pour mieux préserver un instant précieux du passage du temps. En même temps, le silence du tableau qui transforme le rire ou la parole en un mouvement de lèvres suscite un sentiment d'étrangeté, semble mettre un obstacle infranchissable entre la scène, irréelle, et celle qui voit, ici en position de spectatrice de sa propre vision. On se demandera alors dans quel ailleurs cette chambre peut bien se trouver.

10 Figé et animé, le tableau se dédouble aussi lorsqu'il nous donne à voir les choses en transparence. La première femme près de la fenêtre est baignée par la lumière du soleil : « her pale head lit by a summer window, her face slightly shadowed» (33). Cette femme devient dans la suite du paragraphe, «the pale-headed woman » ou « the pale head», et tandis que son visage reste en partie dans l'ombre, c'est l'arrière de sa tête que l'on voit: " [she] could be seen to have the most beautiful slanting ledge of shorn silver and gold from the turn of her skull to the fine neck » (33). Le soleil qui pâlit la jeune femme nous la donne à voir autre, être d'or et de lumière ou être fantomatique. L'emploi du mot « skull » 
attire en tout cas l'attention du lecteur. Tout aussi ambiguë sera la beauté des tasses qui occupent le centre du tableau, "rosily iridescent » : «The lustre glaze streaked the strong pink with cobwebs of blue-grey and white gold " (34). Ce qui donne à l'objet son éclat évoque une fine toile d'araignée. Tout en effaçant la cassure, la vision laisse voir la fragilité des choses en transparence. Les délicates tasses ont beau désormais être intactes, la nature morte célèbre une beauté éphémère qui s'exprime aussi à travers les roses qui envahissent tout le tableau sous différentes formes, pénétrant depuis le jardin jusqu'à l'intérieur de la pièce : "There were leaves coiling into the picture round the outside of the window-frame - a climbing rose, a creeper, what was it?» (33). Le cocon qu'est la chambre rose est déjà ouvert sur l'extérieur - extérieur convoqué d'autre part par l'attente: «they were waiting for someone ». Cette attente ne crée pour l'instant aucune brèche, mais elle met la vision sous le signe d'un suspens qui la dédouble en en différant le sens.

11 L'ailleurs sur lequel s'ouvre le lieu clos est aussi constitué par les tableaux connus que la vision pourra faire surgir en arrière-plan ou plus exactement par la convocation d'une " archipicturalité " ${ }^{3}$ : le tableau évoque ici une nature morte et constitue en même temps une sorte de "conversation piece ». Toile réaliste, il possède par ailleurs un caractère onirique et on pourra y voir l'inscription d'un motif mythologique. Mais là encore on hésitera : les trois jeunes femmes représentent-elles les trois Grâces, ou bien, vu le thème insistant de la couture, et l'effort que doit faire Veronica pour transformer les cheveux gris d'une des femmes, les trois Parques?

\section{Collage et bricolage}

12 En se dédoublant, la vision de Veronica ne se charge pas seulement d'une ambiguïté qui lui permet d'évoquer conjointement une chose et son contraire. La vision, dès lors qu'elle s'épaissit et se feuillette, déplace ce qui serait "premier » dans son élaboration et transforme la transposition en un mouvement qui induit son propre débordement. Se chargeant d'une pluralité potentielle d'« hypo-icônes " ${ }^{4}$, la vision se donne aussi comme résultant de plusieurs transformations simultanées en raison des correspondances multiples qui se dessinent entre les différents tableaux de la vie présente et passée. La chambre de Cambridge offre un cadre dans lequel tel objet pris ça ou là réapparaîtra tel quel ou modifié tandis que tel personnage y sera rajeuni ou habillé avec des vêtements d'une autre époque. En dépit de la tentative d'uniformisation qui s'affiche, la diversité du matériau ne saurait être tout à fait gommée, et certains éléments au sein du tableau demeurent irréductiblement hétérogènes. Des fauteuils, il nous est dit après coup qu'ils jurent dans la vision de Veronica où tout semble par ailleurs évoquer les années vingt: "The chairs in her constructed vision were draped anachronistically in the loose covers they had worn in her brief, half-reluctant glimpse of them » (37). Quelque chose dans la transposition est resté tel quel, résistant à toute adaptation, à toute homogénéisation. L'œuvre montre ses raccords, laisse voir ses coutures et dévoile ainsi une faille ou une défaillance qui trouve un écho dans le deuxième pan de la description, tandis que les vêtements de la mère sont observés de plus près :

Veronica detected in her mother's cream-coloured dress just a touch of awkwardness, her grandmother's ineptness at a trade for which she was not wholly suited, a shoulder out of true, a cuff awry, as so many buttons and cuffs and waistbands had been during the making-do in the time of austerity. This awkwardness in her mother was lovable and vulnerable. (38) 
13 Il y a aussi quelque chose « de travers » dans le tableau de Veronica qui s'apparente à une sorte de patchwork que l'on compose avec ce que l'on a sous la main, un travail de recyclage qui rappelle alors le curieux ouvrage de sa fille Jane: "She appeared to be slicing up a pillow-case and reconstructing it into the curiously formed bandaux and ragribbons that went with certain versions of her hair» (34). Alors que tout, au départ, semble opposer le travail silencieux de Veronica et l'activité quelque peu sauvage de sa fille, c'est en définitive à une même forme de bricolage qu'elles se livrent l'une et l'autre. Loin de simplement recoller les morceaux, la transposition met en pièces autant qu'elle reconstruit, arrachant ça et là des objets à leur support initial pour les juxtaposer et recomposer un tout. Dans ce recyclage, cet art qui consiste à « faire avec » (" make do »), on pourra voir une forme de dépassement : loin de se laisser absorber mélancoliquement par un objet premier, Veronica l'intègre, l'absorbe dans une vision paisible où l'attachement supporte la fracture, et où la continuité est assurée au prix du discontinu.

L'hétérogénéité qui s'affirme au sein de la vision ne résulte toutefois pas seulement de la tentative de faire coller, faute de mieux, les choses ensemble. Tout en relevant d'une manipulation consciente, la vision nous est donnée comme échappant à toute stricte programmation. La chambre avec son atmosphère intime est le lieu dont on reste coupé, exclu, alors même qu'on est en train de le créer de toutes pièces. Si Veronica semble parvenir à une sorte d'arrangement avec les morts, le fait qu'elle se retrouve en même temps spectatrice de sa propre composition ouvre une brèche qui empêche cet arrangement d'être tout à fait définitif. La rêverie éveillée par laquelle le sujet se bâtit un petit scénario commode semble bien emprunter ici, comme le suggère Freud, certaines de ses caractéristiques au rêve.

\section{Indétermination}

15 Lieu d'une mise en scène, la chambre est en même temps une "Autre scène » où, à côté des juxtapositions incongrues, l'étrangeté de la vision tient à son caractère instable, flottant et en certains points opaques. La chambre est un espace qui comme le rêve ignore la contradiction: lieu où la toile figée peut s'animer, lieu où l'on voit les personnages parler ou rire en silence, c'est aussi un endroit qui, pour n'être jamais tout à fait le même, est pourtant considéré comme étant identique à lui-même. Ainsi la description initiale de la scène la pose comme étant unique, telle un tableau, tout en insérant progressivement des adverbes fréquentatifs comme "sometimes ", «always », ou « never »: " Sometimes she saw it burning brightly, but mostly it was dark, because it was summer outside, and through the window, between the rosy chintz curtains, there was the unchanging college garden... » (34). La fusion du singulatif et de l'itératif contribue à faire de la chambre un espace indéterminé, intemporel non en ce qu'il serait négation du temps qui passe, mais dans la mesure où il ne relève pas d'une logique temporelle. Comme le signale Freud, «le rêve n'exprime jamais l'alternative "ou bien - ou bien", mais il recueille ces deux termes comme également justifiés dans la même corrélation $»^{5}$.

16 L'indétermination se traduit aussi par la présence de zones d'ombre dans le tableau: «There was a dark corner containing a piece of furniture she had never managed to see at all - a wardrobe ?» (34). Ce flou se retrouve également en un lieu plus central : l'un des trois personnages féminins n'est pas du tout mentionné dans le deuxième pan de la description et ne fait l'objet que d'une phrase dans le tableau d'ouverture: «One dark 
woman, in a chair, had long hair, knotted in the nape of the neck » (33). Six femmes et quatre générations différentes sont mentionnées au cours de la nouvelle. Cette troisième femme peut être l'une ou plusieurs d'entre elles. S'il s'agit de Veronica, elle nous tourne le dos, résolument spectatrice à l'intérieur comme à l'extérieur du tableau.

Mais l'indétermination principale porte sur le personnage qui apparait au final en point focal du tableau, ce "him» qui oriente soudain la vision au prix d'un nouveau déplacement qui désoriente le lecteur. À la première lecture, cette irruption fugitive d'un inconnu en marge du tableau produit un véritable effet de débordement; elle dessine une ligne de fuite plus qu'un point d'arrêt, une sortie du cadre qui a été soigneusement posé. Cette présence ultime met au premier plan un signifiant qui avait pu passer relativement inaperçu, celui de l'attente: "Her mother raised her pale head expectantly" (38). La scène intime se transforme momentanément en une sorte d'annonciation: pour celle qui est décrite comme «expecting », celui qui entre par la porte pourrait être porteur de la promesse d'une forme de naissance. Veronica, quant à elle, ne verrait-elle pas son patient travail récompensé par cette apparition finale qui s'affiche sur sa toile imaginaire? L'onomastique peut alors fonctionner pleinement et le personnage peut être identifié à la Véronique pour qui Jésus aurait fait miraculeusement apparaître son image sur un drap. En même temps, la vision fugitive s'évanouit aussitôt ; le texte enregistre un mouvement presque simultané d'apparition et de disparition. Le tableau de Veronica ne saurait devenir lui-même "une Véronique », comme la toile qui aurait conservé l'image du Christ.

L'apparition de celui qui vient répondre à l'attente de la mère produit simultanément un effet de bouclage et de relance. L'attention de Veronica se voit déplacée vers un nouvel objet qui finit par éclipser l'objet premier : la mère qui sert de support et de médiation au regard de la fille disparaît à la fin de la nouvelle et Veronica se retrouve seule face au jeune homme souriant. La satisfaction de la mère (et en même temps son silence) a, semble-t-il, un prix pour celle qui reste dans l'ombre: elle fait resurgir un fantôme qui signale peut-être la résistance que rencontre le travail de deuil. Le revenant qui apparaît et disparaît dans un sourire se dérobe ici à plus d'un égard. Non seulement l'évanouissement de la vision condamne le texte au recommencement, mais l'identité de ce « him » restera flottante, même si tout peut laisser à penser que l'homme en question n'est autre que le père de Veronica. Il est intéressant de noter que le sourire, seul trait physique marquant qui permette d'identifier l'inconnu ${ }^{6}$ nous est donné comme la marque d'une singularité qu'il ne détient pourtant pas en propre : le sourire a survécu à l'homme en question, il s'est déplacé et peut réapparaître à tout instant sur la bouche d'une autre. En mettant la fin du texte sous le signe de la ressemblance et de la duplication, la nouvelle nous empêche de dissiper totalement le flou et d'attribuer trop vite une identité à ce fantôme ${ }^{7}$.

Ce qui importe est d'ailleurs sans doute moins l'identité du « him » que la différence ou l'écart qu'il produit, le mouvement de glissement et de substitution potentiellement infini qu'il instaure dès lors qu'il déstabilise la lecture. Le pronom introduit un blanc dans le texte du fait même qu'il reprend ou vient à la place d'un nom qui n'a jamais été prononcé, qui n'a, strictement parlant, aucun antécédent. Cet anaphorique qui, pour citer Pierre Tibi, « postule un avant-texte fantôme » ${ }^{8}$ est courant en début de nouvelle. Il l'est moins en clôture. L'attente, ce sera alors pour le lecteur l'attente d'une interprétation qui pourrait totalement résorber ce vide. Le " him » met par ailleurs en évidence l'existence d'un autre écart ou d'une autre tension dans la nouvelle; car le lecteur peut avoir 
l'impression d'être en reste par rapport au personnage qui, lui, voit : « Veronica saw him smile ». La focalisation interne suggère toutefois que le « him » est « him » pour Veronica elle-même : celui qui n'a pas besoin d'être nommé, ou peut-être celui qui ne doit pas ou ne peut pas être nommé. L'anaphorique sans antécédent a alors aussi une valeur déictique : il permet de montrer, de pointer l'existence de celui dont la singularité ne peut être explicitée. On peut dès lors se demander si le texte est effectivement en reste par rapport à la vision ou, au contraire, s'il ne parvient pas à faire ressortir ce qui dans la vision même serait de l'ordre de l'invisible ou de l'irreprésentable.

\section{L'écriture du tableau}

Cette fin qui nous renvoie au début nous amène alors aussi à relire la nouvelle en nous interrogeant sur la dynamique qu'elle instaure entre le visuel et le textuel. Le texte se présente dans un premier temps comme un travail de transcription d'une image non seulement fictive mais virtuelle : l'image advient donc à l'existence par l'écriture, elle se compose par le texte et simultanément se décompose en texte. Elle apparaît et disparait comme image dans le même mouvement, sans avoir jamais pu exister comme image en tant que telle. Le travail de deuil dans la nouvelle, travail simultané de surinvestissement et de détachement, ce pourrait donc être aussi cet effort de la part de celle qui écrit pour susciter l'image par le texte tout en acceptant de voir l'image transformée en texte. Il est toutefois différentes façons d'envisager cette transformation. S'agit-il d'« apprivoiser " l'image, de renoncer à son pouvoir de capture ou de fascination, d'instaurer une déprise imaginaire en déployant la vision dans un espace tout entier signifiant? Ou s'agit-il de transcrire la force même du visuel, de faire passer dans la langue ce qui ne relève pas de la langue?

21 Il peut sembler que chacun des pans de la nouvelle fonctionne différemment, la description initiale s'attachant à décomposer, à disséquer, à mettre à plat, alors que la vision finale marque la place d'un objet qui se dérobe aux mots avant même de se dérober à la vue. Le fait que le patient montage puisse fonctionner comme un dispositif permettant l'apparition finale suggère que ce sont peut-être essentiellement les limites du découpage et de l'interprétation qu'il s'agit de souligner. Par ailleurs, la description du début est on ne peut plus ambiguë et ne se donne pas simplement comme une conversion du visuel en textuel, mais davantage comme une mise en tension du texte et de l'image qui contribue à rendre tout à fait incertaine la nature de l'objet qui en résulte. La mise en place de la scène imaginaire entraîne en effet le lecteur dans un parcours déroutant qui l'amène sans cesse à réviser ses impressions. D'un côté il semble que la description soit bien une transcription et se mette au service d'une vision qui lui préexiste et dont elle reste en deçà ; de l'autre cette vision s'affirme progressivement comme engendrée dans l'instant même, dans un mouvement où l'effort pour voir et l'effort pour décrire ne peuvent être clairement dissociés. En outre, si le texte nous offre une parfaite illustration de la tentative de "peindre avec les mots ", il interroge aussi le sens possible d'une telle expression.

Le début de la nouvelle masque non seulement le fait que la scène décrite est une construction imaginaire, il masque aussi le fait que cette scène émane d'un regard particulier (Veronica n'est mentionnée qu'à la fin du premier paragraphe), ou même d'un regard tout court. Par cet effacement, le texte simule la force hallucinatoire de la vision fantasmatique qui s'impose sans médiation et semble nous mettre simplement en 
présence des choses. Un premier décrochage consiste à faire apparaître la scène comme telle, à la présenter comme objet perçu par un regard ("this could be seen»). La description semble alors rivaliser avec l'image : extrêmement précise et concrète, elle est repérage méthodique, notation de places, de lignes, de formes, de matières et de couleurs. Un autre décrochage se produit au moment où Veronica, introduite pour la première fois, est posée comme spectatrice passive de cette scène qui, tout en s'imposant à elle, relève d'un artifice : à propos des cheveux de la troisième femme, il est dit : "she had to resist seeing it as she had always known it, pepper and salt» (33). Dès lors, tandis que la description se poursuit tout aussi soignée, le texte donne moins l'impression de transcrire les choses que de les créer dans un effort sans lequel la vision n'existerait pas. Le texte qui tente de rejoindre la vision ne fixe pas ce qui est déjà là, visible, il sort les choses de l'ombre, il les fait advenir à l'existence : chaque phrase sera ainsi comme le geste d'un peintre donnant naissance à un objet peut-être déjà vu mais en même temps entièrement nouveau. Les deux dernières phrases de la description confirment cette impression, et jettent pourtant le lecteur dans la confusion: "She mostly saw the flowers as roses, though many of them, looked at more closely were hybrid or imaginary creations. She was overdoing the pink» (34).

23 Cette dernière phrase crée un effet de rupture brutal d'abord parce que la vision s'apparente tout d'un coup à un vrai tableau ; le lecteur se rend compte qu'il n'avait perçu le «tableau » brossé jusque-là que de façon métaphorique. En outre, au moment même où ce tableau est posé comme objet autonome et vision personnelle ( «She mostly saw the flowers as roses»), il se voit subordonné à une autre vision qui vient renforcer la complexité de la composition («though many of them, looked at more closely were hybrid or imaginary creations »). L'étrangeté de la phrase tient au fait que l'on puisse examiner « de près » des créations tout à fait imaginaires - on se demandera à ce point si le fantasme de Veronica ne s'inspire pas aussi d'un tableau réel (un Matisse par exemple). C'est alors tout le texte qui semble relever d'un phénomène d'hybridation dont il est difficile de rendre compte précisément: dans une certaine mesure, la vision de Veronica peut se concevoir comme obéissant aux lois d'une langue qui tient du rébus - hiéroglyphe d'autant plus insaisissable qu'il ne constitue pas une langue spécifique, mais une langue forgée au gré des circonstances. La description serait alors une tentative d'inscrire par le texte ce qui n'est fondamentalement qu'un autre texte, un scénario fantasmatique que la vision rend ou aura rendu figurable. Mais l'allusion appuyée à la peinture est ambiguë : si le tableau peut-être perçu comme un lieu de condensation du sens, son recours signale aussi peut-être que la vision ne saurait se résumer à un texte et qu'il y a en elle quelque chose qui échappe à l'ordre de la signification.

La mise au premier plan de la couleur fait tout particulièrement ressortir la tension qui s'instaure entre le visuel et le textuel. Face à la prolifération du rose sur le tableau, on pourra en effet se demander à quel impératif la présente composition obéit. Outre la couleur rose, le tableau voit en effet les roses se multiplier sous diverses formes : « floppy roses", "rosebeds", "a climbing rose». Le langage semble alors moins traduire la propagation de la couleur que la faciliter grâce au double statut grammatical du mot qui fait des roses de Veronica elles-mêmes des « créations hybrides ». Les variations lexicales ou grammaticales qui font surgir « rosy » et " rosily » à côté de " rose-coloured » ne font peut-être que rendre la façon dont la couleur envahit tout le tableau, mais elles font aussi inévitablement surgir certaines significations toutes faites: on connait l'emploi métaphorique de «rosy» comme celui de «rose-coloured». La préférence qui est 
accordée dans le titre à l'adjectif composé «rose-coloured» répond sans doute à un impératif phonique et rythmique; en même temps, le choix de l'adjectif composé participe d'une tendance générale dans la nouvelle qui consiste à évoquer la couleur à travers un référent précis. Les robes des trois femmes sont décrites ainsi : « one olive, one russet [...], one, belonging to the pale head, a clotted cream or blanket-wool colour » (33). Le thé qui est versé dans les tasses roses est du thé ambre (" amber tea »,38). S'il est d'un côté très tentant d'« interpréter " la couleur et de l'astreindre à une logique signifiante, la description cherche aussi, semble-t-il, à rendre la matérialité de la vision par des associations qui ne sont pas réductibles à l'interprétation. La transformation soudaine de la vision en ce qui serait un vrai tableau dans « She was overdoing the pink » vient peutêtre en réaction contre la possibilité d'une dérive métaphorique que l'utilisation même du mot « pink » interrompt en nous donnant à voir le « rose » comme de la peinture rose. À ce point, c'est en faisant jouer la dénotation contre la connotation que la description peut dresser le tableau contre le texte. Mais la résistance du texte à l'interprétation passe aussi au final par la constitution d'un objet qui ne rentre dans aucune catégorie, objet hybride, insaisissable, voire impossible.

Dès le début de la nouvelle, les mots semblent donc bien chargés de rendre l'emprise du visuel autant que de permettre une déprise imaginaire. Grâce à la typographie qui détache les unes après les autres les séquences de la vie présente et passée, irrésistiblement vouées à se transformer en tableaux, toute la nouvelle se présente comme une succession de cadres. La brièveté du texte facilite la mise en évidence d'une logique signifiante très serrée qui passe par la mise en correspondance de ces cadres. Mais le cadre a aussi pour effet d'isoler, de détacher, de constituer un espace d'intensité émotionnelle ou pulsionnelle. Il manifeste une capture imaginaire, qui, comme le suggère Barthes, peut aussi bien passer par la voix que par l'image. Dans le chapitre « Ravissement » du Fragment d'un Discours Amoureux, Barthes écrit :

Toujours visuel, le tableau ? Il peut être sonore, le cerne peut être langagier : je puis tomber amoureux d'une phrase qui m'est dite : et non seulement parce qu'elle me dit quelque chose qui vient toucher mon désir, mais à cause de son tour (de son cerne) syntaxique, qui va m'habiter comme un souvenir.'

C'est bien aussi une phrase qui vient faire tableau quand Veronica est sur le point de prononcer les mots de sa propre mère «how could you »: " And abruptly remembered her own mother's voice in the 1950's, unrestrained, wailing, interminable, how could you, how could you, and saw briefly the pair of them [...] ». L'italique fait ressortir ce qui serait une intonation particulière, met en avant l'inflexion singulière de la phrase : le texte se charge alors de nous donner à voir ce qu'il ne peut nous faire entendre. C'est à nouveau cette singularité qui triomphe dans le tableau final lorsque paraît le jeune homme qui se résume pratiquement à un sourire. En même temps que ce sourire surgit quelque chose de tout aussi indéfinissable: la ressemblance physique ou peut-être encore ce que Barthes, dans LaChambre Claire, appelle "l'air». L'air d'un visage, dit Barthes, est « indécomposable ", « chose exorbitante qui induit du corps à l'âme " ${ }^{10}$. À ce point le texte fuit, et ce faisant creuse la vision : le « him » entoure l'apparition du jeune homme d'un silence qui en prolonge l'impact. Tout en sonnant un divorce forcé, la fin de la nouvelle fait émerger un point de convergence : les mots traduisent ici l'adhérence ou l'emprise de l'image en cessant d'essayer de la faire voir pour la faire entendre. Pour parvenir à conserver la force de l'image, il faut en quelque sorte rompre aussi avec elle, ne plus tenter de la décrire mais de l'écrire. Ce n'est alors qu'en élaborant un effet propre à 
l'écriture que pourra être figuré un pouvoir propre à l'image. Le travail de deuil dans la nouvelle pourrait être aussi de réaliser ce passage de la description à l'écriture.

Les deux pans du tableau de "Rose-Coloured Teacups » marquent deux temps que l'on peut rapprocher de ces deux façons de regarder l'image que Barthes distingue à travers le studium et le punctum. Le premier plan nous invite à observer studieusement l'image, à la composer, alors que le second pan voit l'irruption de ce « supplément » qui « vient casser (ou scander) le studium $»^{11}$. Le détail qui apparaît en marge est brutal, déconcertant, il arrête l'interprétation et en même temps prolonge indéfiniment la lecture : «Si fulgurant qu'il soit, le punctum a, plus ou moins virtuellement, une force d'expansion $»^{12}$. La référence à la Chambre Claire, qui prend pour objet l'image photographique, peut être également pertinente si l'on voit dans l'apparition finale " une image révélée », « sortie ", « montée », « exprimée » [...] par l'action de la lumière » ${ }^{13}$, ou encore « une émanation du réel passé : une magie, non un art $\aleph^{14}$. La vision de Veronica relèverait de cette « hallucination tempérée » dont parle Barthes, « fausse au niveau de la perception, vraie au niveau du temps » : « d'un côté "ce n'est pas là", de l'autre "mais cela a bien été" »"15.

L'image marquerait la résistance sinon d'un référent du moins d'un réel ${ }^{16}$ qui ici aussi ferait que « rien, en elle, ne peut transformer le chagrin en deuil $»^{17}$. Les deux pans du tableau mettent également côte à côte deux formes d'arrêt ou de stase: l'effet d'«immobilité vive $»^{18}$ produit par la vision finale vient bousculer l'équilibre et le compromis qui semblent trouvés dans l'instant suspendu que présente la nature morte. Alors que l'espace de la chambre semblait pouvoir tenir et contenir à la fois la vie et la mort, la porte qui s'ouvre introduit avec le « him » un «champ aveugle » ${ }^{19}$, fait surgir le fantôme qui fonde et mine le patient travail de composition de Veronica et la pousse à faire et à défaire sans cesse son ouvrage. Si la nouvelle s'arrête, contrairement au fantasme, l'écriture semble moins se séparer de la vision que souligner à travers elle sa propre limite, son impossibilité de se clore alors même qu'elle cesse. Le texte ne témoigne pas d'un passage à l'écriture qui évacuerait une bonne fois pour toutes fantômes et fantasmes ; le détachement que manifeste l'existence même du texte permet de continuer à dire l'emprise. La façon incertaine et hypothétique selon laquelle texte et image se rencontrent et s'imbriquent nous amène ici à voir la transposition comme un processus d'hybridation qui fait ressortir en quoi le texte ou l'image ne sont jamais identiques à eux-mêmes, jamais clos sur eux-mêmes. Les mettre en correspondance est une façon de mieux traquer le reste qui travaille chacun d'eux et qui fait de l'œuvre un moment ou une étape dans un processus jamais achevé. La nouvelle qui suit, "The July Ghost », marque bien cette nécessité de remettre sans cesse l'ouvrage sur le métier.

\section{BIBLIOGRAPHIE}

A.S. Byatt. « Rose-Coloured Teacups ». Sugar and Other Stories (1987). London: Vintage, 1995.

Barthes, Roland. Fragment d'un discours amoureux. Paris : Seuil, 1977.

Barthes, Roland. La Chambre Claire. Paris : Gallimard, Seuil, 1980. 
Freud, Sigmund. Sur le rêve (1901). Paris : Gallimard, 1988.

Barthes, Roland. « Deuil et Mélancolie » (1917). Cuuvres Complètes, volume XIII. Paris : PUF, 1988.

Louvel, Liliane. L'Æil du texte. Toulouse : PU du Mirail, 1998.

Tibi, Pierre. « La nouvelle : essai de définition d'un genre ». Cahiers de l'Université de Perpignan 4 (printemps 1988).

\section{NOTES}

1. A.S. Byatt, « Rose-Coloured Teacups », Sugar and Other Stories (1987), London: Vintage, 1995.

2. Sigmund Freud, « Deuil et Mélancolie », (1917), Euvres Complètes, volume XIII, Paris : PUF, 1988, 263.

3. Le terme est emprunté à Liliane Louvel dans L'CFil du texte, Toulouse : PU du Mirail, 1998.

4. Ibid.

5. Sigmund Freud, Sur le rêve (1901), Paris : Gallimard, 1988, 95.

6. À la relecture, on rapprochera en effet la description finale « Veronica saw him smile with the wide and shapely smile that had just reappeared, deprecating and casual, on Jane's different, darker face » de la description physique de Jane : "She had her father's big black eyes, outlined in khol, and Veronica's father's wide, shapely mouth».

7. Dans la préface et la postface de Sucre, traduction française de trois des nouvelles du recueil original, Byatt insiste sur le travail de deuil qu'elle a mené à travers l'écriture de ce livre. Elle évoque la disparition de sa mère et de son père, mais aussi la mort brutale de son fils de onze ans, survenue en 1972. Il est frappant de voir comment le sourire qui surgit à la fin de "RoseColoured Teacups » réapparaît d'une certaine façon dans la nouvelle qui suit immédiatement, «The July Ghost ». Dans ce texte, le personnage principal est le fantôme d'un enfant mort qui arbore un sourire radieux mais se dérobe au regard de sa mère. Quoique aucun fils ne soit mentionné dans «Rose-Coloured Teacups", la proximité immédiate de ce fantôme de juillet qu'aucun mur n'arrête laisse se profiler au moins une autre figure à côté de celle du père rajeuni. Le caractère onirique de la vision renforce la possibilité de voir dans le jeune homme une figure composite.

8. Pierre Tibi, «La nouvelle : essai de définition d'un genre ", Cahiers de l'Université de Perpignan 4 (printemps 1988) : 22 .

9. Roland Barthes, Fragment d'un discours amoureux, Paris : Seuil, 1977, 227.

10. Roland Barthes, La Chambre Claire, Paris : Gallimard, Seuil, 1980, 167.

11. Ibid., 48.

12. Ibid., 74 .

13. Ibid., 127.

14. Ibid., 138.

15. Ibid., 177.

16. On peut en effet invoquer la catégorie du réel autrement qu'à travers le référent auquel Barthes fait appel dans La Chambre Claire - en pensant notamment au réel que met en jeu la pulsion.

17. Ibid., 141.

18. Ibid., 81.

19. Ibid., 90. 
INDEX

oeuvrecitee Rose-Coloured Teacups 